\title{
Danos da Morte Súbita dos Citros sobre a Produção de Laranja
}

\author{
Renato B. Bassanezi ${ }^{1}$, Luiz A. Busato ${ }^{1}$, André L. Sanches ${ }^{1}$ \& José C. Barbosa ${ }^{2}$ \\ ${ }^{1}$ Departamento Científico, Fundecitrus, CEP 14807-040, Araraquara, SP, fax: (016) 3301-7032, e-mail: \\ rbbassanezi@fundecitrus.com.br; 'Departamento de Ciências Exatas, Faculdade de Ciências Agrárias e Veterinárias, \\ Universidade Estadual Paulista “Júlio de Mesquita Filho”, CEP 14884-900, Jaboticabal, SP, fax: (016) 3202-4275, \\ e-mail: jcbarbosa@fcav.unesp.br
}

(Aceito para publicação em 23/06/2005)

Autor para correspondência: Renato Beozzo Bassanezi

BASSANEZI, R.B., BUSATO, L.A., SANCHES, A.L. \& BARBOSA, J.C. Danos da Morte Súbita dos Citros sobre a produção de laranja. Fitopatologia Brasileira 30:497-503. 2005.

\section{RESUMO}

A Morte Súbita dos Citros (MSC) afeta laranjeiras doces (Citrus sinensis) e algumas tangerineiras (Citrus reticulata) enxertadas em limoeiro ‘Cravo' (Citrus limonia) no norte do Estado de São Paulo e sul do Triângulo Mineiro. O progresso da doença nos pomares têm causado grande preocupação para o agronegócio citrícola. Para caracterizar e quantificar os danos causados pela MSC, a produção de frutos (peso total e número de frutos por planta e tamanho dos frutos) foi avaliada em quatro talhões para cada combinação variedade ('Hamlin', 'Pêra', 'Natal' e 'Valência') / classe de idade (três a cinco anos, seis a dez anos e 11 a 15 anos). Em cada talhão, as plantas foram classificadas de acordo com a severidade de MSC ( 0 = sadia, 1 = sintomas iniciais e 2 = sintomas severos). Para cada nível de severidade foram colhidas dez plantas escolhidas ao acaso. Os danos foram caracterizados pela redução do peso total de frutos por planta (28\% e $50 \%$ para o nível 1 e 2 , respectivamente), do número total de frutos por planta (12\% e $26 \%$ para o nível 1 e 2, respectivamente) e do tamanho do fruto (22\% e $41 \%$ para nível 1 e 2, respectivamente). As variedades de laranja não diferiram em relação à porcentagem de redução para todas as variáveis de produção avaliadas. As plantas mais jovens tiveram a maior redução no número total de frutos por planta e as plantas com mais de cinco anos de idade tiveram as maiores reduções no tamanho dos frutos. As classes de idade não diferiram na porcentagem de redução do peso total de frutos por planta.

Palavras-chave adicionais: epidemiologia, avaliação de danos, severidade, etiologia, Citrus sinensis, laranja doce.

\begin{abstract}
Citrus Sudden Death Damages on sweet orange yield

Citrus Sudden Death (CSD) affects sweet oranges (Citrus sinensis) and some mandarins (Citrus reticulata) grafted on Rangpur lime (Citrus limonia) in the northern region of the State of São Paulo and south of the Triângulo Mineiro, Brazil. The progress of the disease in these groves is of great concern to citrus agribusiness. To characterize and quantify the damage caused by CSD, fruit yield (total weight and fruit number by plant, and fruit size) was assessed in four blocks for each combination of sweet orange varieties ('Hamlin', 'Pêra', 'Natal', and 'Valência') and class of age (three to five years, six to ten years, and 11 to 15 years). In each block, trees were classified according to CSD severity $(0=$ healthy, $1=$ initial symptoms, and 2 = severe symptoms). For each severity level, ten trees were selected at random and harvested. Damage was characterized by reduction of total weight of harvested fruit per plant (28\% and 50\% for level 1 and 2 , respectively), total number of fruit per plant (12\% and 26\% for level 1 and 2, respectively), and fruit size ( $22 \%$ and $41 \%$ for level 1 and 2, respectively). There was no difference in percentage of reduction of all evaluated variables of fruit yield among the tested sweet orange varieties. The youngest plants showed a higher reduction in fruit number per plant, while plants older than five years showed a higher reduction in fruit size. There was no difference in percentage of reduction of total weight of fruit per plant among plants of different age classes.
\end{abstract}

Additional keywords: epidemiology, damage evaluation, disease severity, etiology, Citrus sinensis, sweet orange.

\section{INTRODUÇÃO}

A Morte Súbita dos Citros (MSC), descrita pela primeira vez no início de 2001 (Gimenes-Fernandes \& Bassanezi, 2001), é uma doença cujo agente causal, ainda desconhecido, pode ser transmitido pela enxertia de tecidos infetados em plantas sadias (Yamamoto et al., 2003). Acredita-se que a MSC seja semelhante à tristeza dos citros na sua forma de declínio rápido, quando a laranjeira doce [Citrus sinensis (L.) Osbeck] é enxertada sobre a laranjeira ‘Azeda' (Citrus aurantium L.), e que seja causada por um vírus disseminado por um vetor. Esta hipótese é baseada na associação constante das plantas com sintomas de MSC com o Citrus tristeza virus (CTV), pertencente à família Closteroviridae, gênero Closterovirus, e nas semelhanças entre a MSC e a tristeza quanto à sintomatologia (GimenesFernandes \& Bassanezi, 2001) e às alterações ultraestruturais (Román et al., 2004), à velocidade de progresso da doença e à distribuição espacial das plantas afetadas nos talhões (Bassanezi et al., 2003a; Jesus Junior \& Bassanezi, 2004), e por serem doenças de combinação copa/porta-enxerto (Gimenes-Fernandes \& Bassanezi, 2001; Müller et al., 2002),

Fitopatol. bras. 30(5), set - out 2005 
cuja remissão dos sintomas pode ser conseguida pela troca do porta-enxerto pela sub-enxertia com porta-enxertos tolerantes (Bassanezi et al., 2003b).

Por afetar pomares de laranjeiras doces das variedades 'Hamlin', 'Pêra', 'Valência' e 'Natal' enxertadas sobre limoeiro ‘Cravo’ (Citrus limonia Osbeck), que representam 85\% das plantas no Estado de São Paulo (Bassanezi et al., 2003b), a MSC representa séria ameaça à citricultura brasileira, assim como foi a tristeza, que entre 1939 e 1949 destruiu 90\% das plantas existentes em São Paulo (Kimati \& Galli, 1980). Desde sua constatação, o progresso e a disseminação do agente causal da doença nos pomares afetados têm sido bastante rápidos, podendo atingir de 30 a 70\% das plantas em apenas seis meses (Bassanezi et al., 2002; Bassanezi et al., 2003a; Bassanezi et al., 2003b). Entre setembro de 2002 e dezembro de 2003, o número de plantas com sintomas de MSC encontradas no campo, no Estado de São Paulo, passou de 22,1 mil para 44,4 mil e outras 392,2 mil plantas foram erradicadas pela doença no mesmo período. Estima-se que cerca de dois milhões de árvores já tenham sido afetadas pela doença desde sua descoberta em fevereiro de 2001, nos municípios paulistas e mineiros (Fundecitrus, 2004). De dezembro de 2001 a dezembro de 2003, a doença passou de sete municípios no sul do Triângulo Mineiro e no norte do Estado de São Paulo (Libanore et al., 2002) para 30 municípios (Fundecitrus, 2004).

As plantas afetadas apresentam sintomas de definhamento generalizado. Inicialmente, toda a copa da planta afetada apresenta folhas sem brilho e de coloração verde pálida, poucas brotações, ausência de brotações internas e queda de folhas. O sistema radicular apresenta poucas radicelas e grande quantidade de raízes podres e mortas. Os tecidos internos da casca do porta-enxerto abaixo da região da enxertia apresentam coloração amarelada a alaranjada, contrastando com a coloração creme dos tecidos da casca da copa (Gimenes-Fernandes \& Bassanezi, 2001). Estes tecidos do porta-enxerto abaixo da zona de enxertia apresentam redução do tamanho das células do floema, colapso e degradação dos tubos crivados, superprodução e degradação do floema, acúmulo de floema não funcional e invasão do córtex pelo floema velho e não funcional (Román et al., 2004).

Quando ocorre acentuado desequilíbrio entre a demanda de água e sais minerais pela copa e o suprimento fornecido pelo sistema radicular, comum em plantas com alta carga de frutos, a planta entra em colapso e o quadro de morte súbita é observado em poucas semanas após o aparecimento dos primeiros sintomas. Entretanto, na maioria dos casos, ocorre o declínio gradual das plantas por até alguns anos, durante os quais são alternados períodos de declínio com períodos de recuperação até que seja atingido um estado de equilíbrio entre a copa e o sistema radicular. Neste caso, as plantas afetadas se mantêm vivas por mais tempo, porém são pouco produtivas (Bassanezi et al., 2003b). Portanto, os danos causados pela MSC devem ser relacionados muito mais à redução na produtividade das plantas, ou “morte econômica”, que à morte biológica das mesmas.

Até o momento, não há dados sobre a estimativa de danos causados por essa doença sobre a produção de frutos. A quantificação dos danos causados pela MSC, além de mostrar a importância da doença, serve para definir o momento em que o talhão afetado deixa de ser economicamente viável para o citricultor. Desta forma, este trabalho foi desenvolvido para avaliar os danos causados pela MSC na produção de frutos em função da severidade da doença em plantas de diferentes idades das principais variedades de laranjeira doce cultivadas em São Paulo.

\section{MATERIAL E MÉTODOS}

O trabalho foi desenvolvido na safra 2003 em talhões de laranjeira doce sobre limoeiro ‘Cravo' com plantas mostrando diferentes níveis de severidade de sintomas de MSC. Os talhões selecionados eram localizados no município de Colômbia, no norte do Estado de São Paulo, e nos municípios de Planura, Frutal, Comendador Gomes e Uberlândia, no sul do Triângulo Mineiro.

As variedades avaliadas foram as laranjeiras 'Hamlin', 'Pêra', 'Natal' e 'Valência' e as classes de idades de cada variedade foram três a cinco anos; seis a dez anos e 11 a 15 anos. Para cada uma das 12 combinações variedade/ classe de idade foram amostrados quatro talhões (quatro repetições), fazendo um total de 48 talhões avaliados.

Dentro de cada talhão todas as plantas foram classificadas de acordo com a seguinte escala descritiva de severidade de sintomas da MSC estabelecida para este trabalho: a) nível 0 (zero), planta sadia ou aparentemente sadia; b) nível 1, planta com sintomas iniciais da doença, mostrando as folhas da copa sem o brilho e sem a coloração verde-escura característicos de uma planta sadia e também com amarelecimento interno da casca do porta-enxerto abaixo da zona de enxertia; c) nível 2, planta com sintomas severos de MSC, caracterizada, além dos sintomas anteriores, pela desfolha parcial da planta e poucas brotações internas (Figura 1).

Dez plantas em cada nível de severidade foram escolhidas ao acaso e marcadas previamente para a avaliação da produção em cada talhão. Na época da colheita, as plantas selecionadas em cada talhão foram colhidas individualmente. Em cada planta, foram avaliados o peso total dos frutos por planta, o número total de frutos por planta e o número de frutos por sacola de colheita de $27,2 \mathrm{~kg}$, que representa uma medida inversamente proporcional ao tamanho dos frutos.

A avaliação da produção foi feita em cada talhão à medida que se aproximava a época de colheita de cada variedade: 'Hamlin' de junho a julho, 'Pêra' de agosto a novembro, 'Valência' de setembro a novembro e 'Natal' de agosto a outubro.

Para cada combinação variedade/classe de idade realizou-se uma análise de variância com três tratamentos (níveis 0, 1 e 2 de severidade de MSC) e quatro blocos (talhões), de acordo com o delineamento em blocos casualizados. Cada parcela foi constituída por dez plantas 


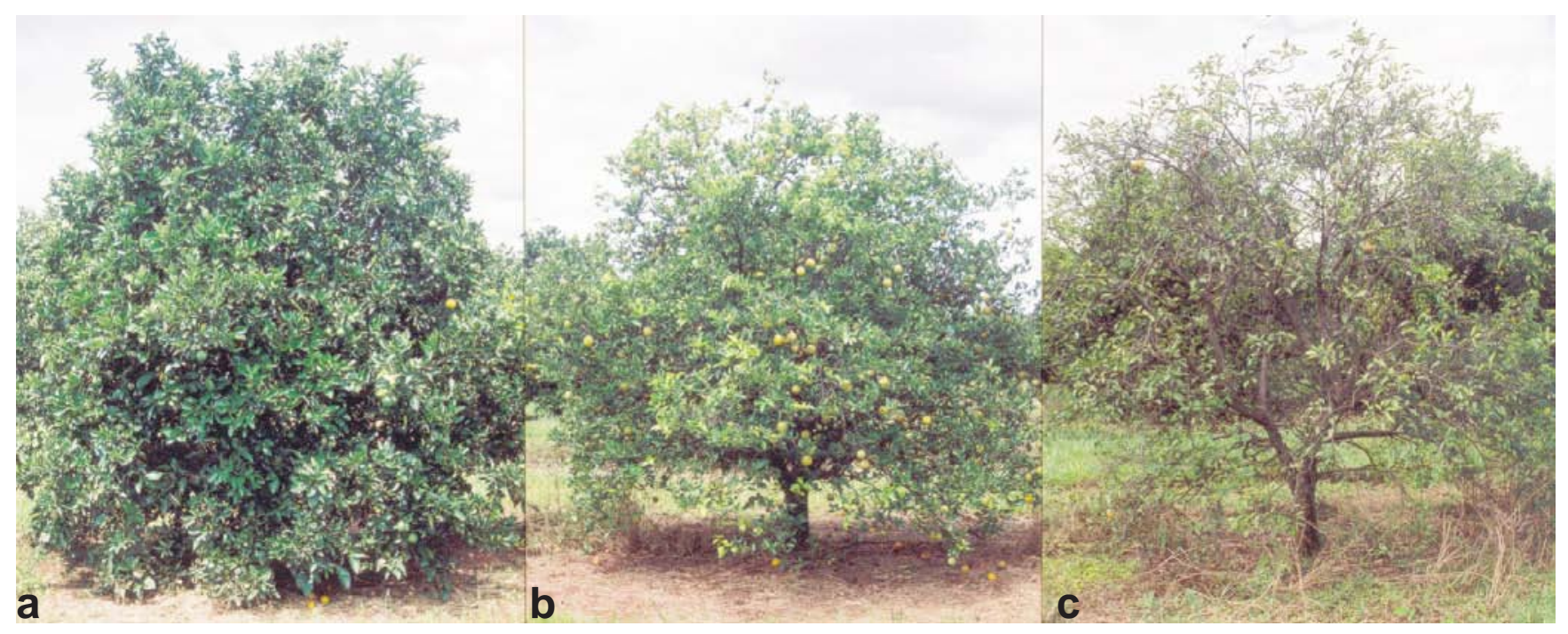

FIG. 1 - Níveis de severidade de Morte Súbita dos Citros (MSC). a) nível 0 - planta sadia ou aparentemente sadia; b) nível 1 - planta com sintomas iniciais, mostrando as folhas da copa sem o brilho e sem a coloração verde-escura característica de uma planta sadia e o amarelecimento interno da casca do porta-enxerto abaixo na zona de enxertia; c) nível 2 - planta com sintomas severos, caracterizada, além dos sintomas anteriores, pela desfolha parcial da planta e poucas brotações internas.

em cada nível de severidade.

As médias dos valores absolutos das variáveis de produção não foram comparadas entre as variedades e classes de idade, pois já se sabe da existência de diferença em termos de produção entre as variedades testadas e que as plantas em diferentes idades produzem mais à medida que vão atingindo maiores idades. Para eliminar essa diferença intrínseca das variedades e idades na produção e permitir a comparação do efeito da MSC nas variáveis de produção entre as variedades de laranja e entre as classes de idade, os dados de peso total de frutos por planta, número total de frutos por planta e número de frutos por sacola de colheita para os níveis 1 e 2 de severidade foram transformados em porcentagem em relação ao nível 0 de severidade, tornando todos os dados relativos às plantas sadias de cada variedade e idade. A partir daí, para cada nível de severidade (1 e 2) realizou-se uma análise de variância com 12 tratamentos (fatorial quatro variedades x três classes de idade) e quatro blocos (talhões) por tratamento, de acordo com o delineamento em blocos casualizados. Como não houve interação significativa para variedade x classe de idade para as variáveis de produção relativas ao nível 0 nos dois níveis de severidade de sintomas de MSC (1 e 2), os efeitos dos fatores variedade e classe de idade não dependem entre si e, portanto foram analisados separadamente.

Quando detectada diferença significativa entre as médias pelo teste $\mathrm{F}$, as mesmas foram comparadas pelo teste de Tukey à $5 \%$ de significância.

\section{RESULTADOS}

Os danos causados pela MSC na produção de frutos de laranja foram caracterizados pela redução do peso total e número total de frutos por planta, e pelo aumento no número de frutos por sacola de colheita de 27,2 kg nas plantas doentes.

A redução do peso total de frutos por planta foi significativa para maioria das combinações variedade/classe de idade estudadas quando se comparam os níveis 1 e 2 em relação ao nível 0 (Tabela 1). Quanto mais severos os sintomas de MSC, maiores foram as reduções no peso total de frutos por planta. Não houve diferença significativa entre variedades para o peso total de frutos por planta e as reduções médias observadas para os níveis 1 e 2 em relação ao nível 0 variaram de 19,14 a 34,53\% (média $=27,59 \%$ ) e de 44,74 a 55,49\% (média $=50,41 \%$ ), respectivamente (Tabela 2). Considerando-se a classe de idade das plantas, as reduções médias observadas em relação ao nível 0 seguiram a mesma proporção, de 25,67 a 28,91\%, para nível 1, e de 49,16 a $51,53 \%$, para o nível 2, não havendo diferenças significativas entre as classes de idade, isto é, não houve uma classe de idade na qual os danos no peso total de frutos fossem mais pronunciados (Tabela 3).

A redução do número total de frutos por planta não foi significativa para a maioria das variedades e classes de idade estudadas quando se comparou o nível 1 com o nível 0 (Tabela 4). Entretanto, quando a planta apresentou nível 2 de MSC, na maioria dos casos, foram observadas diferenças significativas no número total de frutos. As reduções médias observadas em relação ao nível 0 entre as variedades para o número total de frutos por planta nos níveis 1 e 2 variaram de 3,30 a $22,04 \%$ (média $=11,64 \%$ ) e de 15,95 a $36,12 \%$ (média $=25,64 \%$ ), respectivamente (Tabela 2). Novamente, não houve diferença significativa entre variedades. Para as classes de idade, as reduções médias observadas em relação ao nível 0 foram significativamente maiores nas plantas com três a cinco anos (23,52 e 42,03\% para os níveis 1 e 2, 
TABELA 1 - Peso total de frutos por planta (kg) nas combinações variedade de laranjeira (Citrus sinensis)/classe de idade em função dos níveis de severidade de sintomas da Morte Súbita dos Citros (MSC) (média de quatro talhões e dez plantas por talhão)

\begin{tabular}{|c|c|c|c|c|c|c|c|c|c|}
\hline \multicolumn{2}{|c|}{ Combinação } & \multicolumn{6}{|c|}{ "Níveis de severidade de MSC } & \multirow{2}{*}{ Teste $\mathbf{F}^{2}$} & \multirow{2}{*}{$\mathbf{C V}$} \\
\hline Variedade & Idade & $\mathbf{0}$ & & 1 & & 2 & & & \\
\hline 'Hamlin' & 3 a 5 anos & 63,68 & $\mathrm{a}$ & 51,39 & $\mathrm{a}$ & 32,62 & $\mathrm{~b}$ & $9,54 *$ & 32,46 \\
\hline 'Hamlin' & 6 a 10 anos & 148,42 & $\mathrm{a}$ & 127,09 & $\mathrm{a}$ & 81,24 & $\mathrm{~b}$ & $22,54 * *$ & 29,76 \\
\hline 'Hamlin' & 11 a 15 anos & 224,58 & $\mathrm{a}$ & 157,14 & $\mathrm{~b}$ & 95,41 & $\mathrm{c}$ & $34,28 * *$ & 32,03 \\
\hline 'Pêra' & 3 a 5 anos & 81,31 & $\mathrm{a}$ & 42,87 & $\mathrm{~b}$ & 29,78 & $\mathrm{~b}$ & $75,19 *$ & 33,38 \\
\hline 'Pêra' & 6 a 10 anos & 84,25 & a & 67,55 & $a b$ & 53,16 & $\mathrm{~b}$ & $16,26 *$ & 33,07 \\
\hline 'Pêra' & 11 a 15 anos & 84,82 & $\mathrm{a}$ & 64,13 & $\mathrm{~b}$ & 48,16 & $\mathrm{c}$ & $117,34 * *$ & 31,10 \\
\hline 'Natal' & 3 a 5 anos & 66,10 & $\mathrm{a}$ & 47,61 & $a b$ & 34,10 & $\mathrm{~b}$ & $10,22 *$ & 29,52 \\
\hline 'Natal' & 6 a 10 anos & 103,82 & $\mathrm{a}$ & 64,32 & $\mathrm{~b}$ & 39,61 & $\mathrm{~b}$ & $13,20 * *$ & 26,56 \\
\hline 'Natal' & 11 a 15 anos & 131,79 & $\mathrm{a}$ & 78,23 & $\mathrm{~b}$ & 56,14 & $\mathrm{~b}$ & $19,26 * *$ & 32,75 \\
\hline 'Valência' & 3 a 5 anos & 76,56 & $\mathrm{a}$ & 49,01 & $a b$ & 31,89 & $\mathrm{~b}$ & $6,44 *$ & 28,87 \\
\hline 'Valência' & 6 a 10 anos & 142,09 & $\mathrm{a}$ & 86,17 & $\mathrm{~b}$ & 64,39 & $\mathrm{~b}$ & $17,73 * *$ & 27,12 \\
\hline 'Valência' & 11 a 15 anos & 105,20 & $\mathrm{a}$ & 78,89 & $a b$ & 53,07 & $\mathrm{~b}$ & $5,75 *$ & 29,42 \\
\hline
\end{tabular}

${ }^{1}$ Valores com a mesma letra na linha não diferem significativamente entre si pelo Teste de Tukey ao nível de 5\% de probabilidade;

${ }^{2}$ Valor de F significativo ao nível de 5\% (*) ou 1\% (**) de probabilidade.

TABELA 2 - Porcentagem da redução do peso total de frutos por planta de laranjeira doce (Citrus sinensis), da redução do número total de frutos por planta e do aumento do número de frutos por sacola de colheita de $27,2 \mathrm{~kg}$ em relação à plantas sadias (nível 0) em cada nível de severidade de sintoma da Morte Súbita dos Citros (níveis 1 e 2) para as variedades de laranjeira (média de 12 talhões e dez plantas por talhão)

\begin{tabular}{|c|c|c|c|c|c|c|c|c|c|c|}
\hline \multirow{4}{*}{$\begin{array}{c}\text { Níveis de MSC } \\
1\end{array}$} & \multicolumn{10}{|c|}{ Variedades de laranja doce (Citrus sinensis) } \\
\hline & \multicolumn{10}{|c|}{$\%$ de redução no peso total de frutos por planta em relação ao nível $0^{1}$} \\
\hline & 'Hamlin' & \multicolumn{2}{|l|}{ 'Pêra' } & \multicolumn{2}{|c|}{ 'Natal' } & \multicolumn{2}{|c|}{ 'Valência' } & \multirow{2}{*}{$\begin{array}{c}\text { Média } \\
27,59\end{array}$} & \multirow{2}{*}{$\begin{array}{l}\text { Teste } \mathbf{F}^{2} \\
2,28^{\mathrm{NS}}\end{array}$} & \multirow{2}{*}{$\begin{array}{c}\mathbf{C V} \\
20,93\end{array}$} \\
\hline & 19,14 a & 26,57 & $\mathrm{a}$ & 34,53 & $\mathrm{a}$ & 30,62 & $\mathrm{a}$ & & & \\
\hline \multirow[t]{3}{*}{2} & $49,37 \quad \mathrm{a}$ & 44,74 & $\mathrm{a}$ & 55,49 & $\mathrm{a}$ & 51,17 & $\mathrm{a}$ & 50,41 & $0,51^{\mathrm{NS}}$ & 30,77 \\
\hline & \multicolumn{10}{|c|}{$\%$ de redução no número total de frutos por planta em relação ao nível $0^{1}$} \\
\hline & 'Hamlin' & 'Pêra' & & 'Natal & & 'Valência & & Média & Teste $\mathbf{F}^{2}$ & CV \\
\hline 1 & 3,30 a & 6,01 & a & 22,04 & $\mathrm{a}$ & 14,96 & a & 11,64 & $0,27^{\mathrm{NS}}$ & 27,52 \\
\hline \multirow[t]{3}{*}{2} & $23,87 \quad \mathrm{a}$ & 15,95 & a & 36,12 & $\mathrm{a}$ & 25,14 & $\mathrm{a}$ & 25,64 & $0,96^{\mathrm{NS}}$ & 30,87 \\
\hline & \multicolumn{10}{|c|}{ \% de aumento no número de frutos por sacola em relação ao nível $0^{1}$} \\
\hline & 'Hamlin' & 'Pêra' & & 'Natal & & 'Valência & & Média & Teste $^{2}$ & $\mathrm{CV}$ \\
\hline 1 & $20,18 \quad$ a & 28,22 & $\mathrm{a}$ & 16,49 & $\mathrm{a}$ & 22,58 & $\mathrm{a}$ & 21,56 & $0,62^{\mathrm{NS}}$ & 14,27 \\
\hline 2 & 44,49 a & 44,31 & $\mathrm{a}$ & 28,52 & $\mathrm{a}$ & 45,63 & $\mathrm{a}$ & 40,67 & $1,27^{\mathrm{NS}}$ & 19,87 \\
\hline
\end{tabular}

${ }^{1}$ Valores com a mesma letra na linha não diferem significativamente entre si pelo Teste de Tukey ao nível de 5\% de probabilidade;

${ }^{2}$ Valor de F não significativo (NS).

respectivamente), seguidas pelas plantas com 11 a 15 anos (12,18 e 21,37\% para os níveis 1 e 2, respectivamente). Na classe com seis a dez anos as reduções causadas pela MSC no número total de frutos foram pouco pronunciadas $(-0,04$ e 14,56\% para os níveis 1 e 2, respectivamente) (Tabela 3).

Quanto ao número de frutos por sacola de colheita, houve um aumento significativo para a maioria das variedades e classes de idade estudadas quando se comparam os níveis 1 e 2 com o nível 0 (Tabela 5). Quanto mais severos foram os sintomas de MSC, maiores foram os aumentos do número de frutos por sacola, o que significa que o tamanho dos frutos foi menor nas plantas com níveis avançados de MSC, pois em um mesmo volume de sacola couberam mais frutos. Os aumentos médios observados entre as variedades para o número de frutos por sacola nos níveis 1 e 2 variaram de 16,49 a $28,22 \%$ (média $=21,56 \%$ ) e de 28,52 a $45,63 \%$ (média $=40,67 \%$ ), respectivamente (Tabela 2). Não houve, entre as variedades, uma que apresentasse uma redução do tamanho de frutos mais significativa que as demais. Para as classes de idade, não houve redução significativa do tamanho dos frutos nas plantas mais jovens (de três a cinco anos). As reduções no tamanho de frutos foram maiores nas plantas com seis a dez anos, o que significou um aumento do número de frutos por sacola de 35,36 e 65,65\% para os níveis 1 e 2, respectivamente, seguidas pelas plantas com 11 a 15 anos, cujo aumento do número de frutos por sacola foi de 24,46 e $55,79 \%$ para os níveis 1 e 2, respectivamente (Tabela 3). 
TABELA 3 - Porcentagem da redução do peso total de frutos por planta de laranjeira doce (Citrus sinensis) por planta, da redução do número total de frutos por planta e do aumento do número de frutos por sacola de colheita de 27,2 kg em relação à plantas sadias (nível 0) em cada nível de severidade de sintoma da Morte Súbita dos Citros (níveis 1 e 2) para as classes de idade (média de 16 talhões e dez plantas por talhão)

\begin{tabular}{|c|c|c|c|c|c|c|c|c|c|}
\hline \multirow{3}{*}{ Níveis de MSC } & \multicolumn{9}{|c|}{ Classes de idade } \\
\hline & \multicolumn{9}{|c|}{ \% de redução no peso total de frutos por planta em relação ao nível $0^{1}$} \\
\hline & \multicolumn{2}{|c|}{3 a 5 anos } & \multicolumn{2}{|c|}{6 a 10 anos } & \multicolumn{2}{|c|}{11 a 15 anos } & \multirow{3}{*}{$\begin{array}{c}\text { Média } \\
27,59 \\
50,41\end{array}$} & \multirow{3}{*}{$\begin{array}{c}\text { Teste } \mathbf{F}^{2} \\
0,52^{\mathrm{NS}} \\
0,28^{\mathrm{NS}} \\
\end{array}$} & \multirow{2}{*}{$\begin{array}{l}\mathbf{C V} \\
20,93\end{array}$} \\
\hline 1 & 28,20 & a & 25,67 & $\mathrm{a}$ & 28,91 & a & & & \\
\hline \multirow[t]{3}{*}{2} & 51,53 & a & 49,16 & a & 50,61 & a & & & 30,77 \\
\hline & \multicolumn{9}{|c|}{ \% de redução no número total de frutos por planta em relação ao nível $0^{1}$} \\
\hline & \multicolumn{2}{|c|}{3 a 5 anos } & \multicolumn{2}{|c|}{6 a 10 anos } & \multicolumn{2}{|c|}{11 a 15 anos } & Média & Teste $\mathbf{F}^{2}$ & $\mathbf{C V}$ \\
\hline 1 & 23,52 & $\mathrm{~b}$ & $-0,04$ & a & 12,18 & $\mathrm{ab}$ & 11,64 & $4,11 *$ & 27,52 \\
\hline \multirow[t]{3}{*}{2} & 42,03 & $\mathrm{~b}$ & 14,56 & $\mathrm{a}$ & 21,37 & $\mathrm{a}$ & 25,64 & $6,15 * *$ & 30,87 \\
\hline & \multicolumn{9}{|c|}{ \% de aumento no número de frutos por sacola em relação ao nível $0^{1}$} \\
\hline & \multicolumn{2}{|c|}{3 a 5 anos } & \multicolumn{2}{|c|}{6 a 10 anos } & \multicolumn{2}{|c|}{11 a 15 anos } & Média & Teste $\mathbf{F}^{2}$ & $\mathbf{C V}$ \\
\hline 1 & 3,75 & $\mathrm{~b}$ & 35,36 & $\mathrm{a}$ & 24,46 & $\mathrm{a}$ & 21,56 & $10,77 * *$ & 14,27 \\
\hline 2 & $-2,12$ & $\mathrm{~b}$ & 65,65 & $\mathrm{a}$ & 55,79 & $\mathrm{a}$ & 40,67 & $23,95 * *$ & 19,87 \\
\hline
\end{tabular}

${ }^{1}$ Valores com a mesma letra na linha não diferem significativamente entre si pelo Teste de Tukey ao nível de 5\% de probabilidade;

${ }^{2}$ Valor de F não significativo (NS), significativo ao nível de $5 \%(*)$ ou $1 \%(* *)$ de probabilidade.

TABELA 4 - Número total de frutos por planta nas combinações variedade de laranjeira (Citrus sinensis)/classe de idade em função dos níveis de severidade de sintomas da Morte Súbita dos Citros (média de quatro talhões e dez plantas por talhão)

\begin{tabular}{|c|c|c|c|c|c|c|c|c|c|}
\hline \multicolumn{2}{|c|}{ Combinação } & \multicolumn{6}{|c|}{ 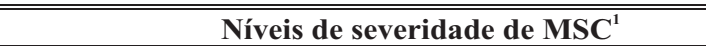 } & \multirow{2}{*}{ Teste $\mathbf{F}^{2}$} & \multirow{2}{*}{$\mathbf{C V}$} \\
\hline Variedade & Idade & $\mathbf{0}$ & & 1 & & 2 & & & \\
\hline 'Hamlin' & 3 a 5 anos & 469,49 & a & 403,10 & $\mathrm{a}$ & 289,33 & $\mathrm{~b}$ & $5,60 *$ & 39,15 \\
\hline 'Hamlin’' & 6 a 10 anos & 923,90 & a & 1056,69 & a & 1019,17 & $\mathrm{a}$ & $0,62^{\mathrm{NS}}$ & 39,42 \\
\hline 'Hamlin’' & 11 a 15 anos & 2004,24 & a & 1584,67 & $\mathrm{ab}$ & 1083,67 & $\mathrm{~b}$ & $11,15 * *$ & 36,76 \\
\hline 'Pêra' & 3 a 5 anos & 593,60 & a & 382,48 & $\mathrm{ab}$ & 294,51 & $\mathrm{~b}$ & $31,77 *$ & 32,68 \\
\hline 'Pêra' & 6 a 10 anos & 448,96 & $\mathrm{a}$ & 475,76 & $\mathrm{a}$ & 404,57 & $\mathrm{a}$ & $2,04^{\mathrm{NS}}$ & 34,47 \\
\hline 'Pêra' & 11 a 15 anos & 650,47 & $\mathrm{a}$ & 616,39 & $\mathrm{a}$ & 608,69 & $\mathrm{a}$ & $0,25^{\mathrm{NS}}$ & 35,77 \\
\hline 'Natal' & 3 a 5 anos & 469,46 & $\mathrm{a}$ & 358,28 & $\mathrm{ab}$ & 299,41 & $\mathrm{~b}$ & $5,35^{*}$ & 34,90 \\
\hline 'Natal' & 6 a 10 anos & 815,78 & $\mathrm{a}$ & 628,60 & $\mathrm{a}$ & 465,75 & $\mathrm{a}$ & 4,53 NS & 28,78 \\
\hline 'Natal' & 11 a 15 anos & 908,71 & $\mathrm{a}$ & 690,06 & $\mathrm{~b}$ & 617,62 & $\mathrm{~b}$ & $12,13 * *$ & 37,12 \\
\hline ‘Valência' & 3 a 5 anos & 489,63 & $\mathrm{a}$ & 323,02 & $\mathrm{a}$ & 227,94 & $\mathrm{a}$ & 3,72 NS & 34,70 \\
\hline 'Valência' & 6 a 10 anos & 996,41 & $\mathrm{a}$ & 796,05 & a & 718,45 & $\mathrm{a}$ & $1,33^{\mathrm{NS}}$ & 32,77 \\
\hline 'Valência' & 11 a 15 anos & 820,87 & $\mathrm{a}$ & 768,66 & $\mathrm{a}$ & 703,65 & $\mathrm{a}$ & $0,35^{\mathrm{NS}}$ & 33,02 \\
\hline
\end{tabular}

${ }^{1}$ Valores com a mesma letra na linha não diferem significativamente entre si pelo Teste de Tukey ao nível de $5 \%$ de probabilidade;

${ }^{2}$ Valor de F não significativo (NS), significativo ao nível de $5 \%(*)$ ou $1 \%(* *)$ de probabilidade.

\section{DISCUSSÃO}

Quanto mais severos os sintomas da doença, maiores foram as diferenças de peso, número e tamanho dos frutos colhidos. Plantas com sintomas inicias de MSC apresentaram uma produção, em média, 27,59\%, 11,64\% e 21,56\% menor que a das plantas sadias em termos de peso total de frutos por planta, número total de frutos por planta e tamanho de fruto, respectivamente. Plantas com sintomas severos de MSC reduziram, em média, $50,41 \%$ do peso total de frutos por planta, 25,64\% do número total de frutos por planta e 40,67\% do tamanho de frutos em relação às plantas sadias.

Estes danos significativos causados pela MSC na produção de frutos de laranja foram decorrentes da evolução de todo um quadro sintomatológico desenvolvido após a infeção da planta. De acordo com Román et al. (2004), as primeiras alterações observadas nas plantas com MSC estão relacionadas ao mal funcionamento e degradação das células do floema nos tecidos do porta-enxerto de limão 'Cravo'. Em plantas afetadas, os tecidos do porta-enxerto abaixo da zona de enxertia apresentam redução do tamanho das células do floema, colapso e degradação dos tubos crivados, degradação do floema, acúmulo de floema não funcional e invasão do córtex pelo floema não funcional velho. Em resposta à degradação das células do floema, o câmbio vascular promove a superprodução de novas células de floema, que posteriormente também são degradadas (Román et al., 2004). Este processo contínuo de degradação e síntese 
TABELA 5 - Número de frutos por sacola de colheita de 27,2 kg nas combinações variedade de laranjeira (Citrus sinensis)/classe de idade em função dos níveis de severidade de sintomas da Morte Súbita dos Citros (média de quatro talhões e dez plantas por talhão)

\begin{tabular}{|c|c|c|c|c|c|c|c|c|c|}
\hline \multicolumn{2}{|c|}{ Combinação } & \multicolumn{6}{|c|}{ 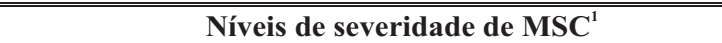 } & \multirow{2}{*}{ Teste $\mathbf{F}^{2}$} & \multirow{2}{*}{$\mathbf{C V}$} \\
\hline Variedade & Idade & $\mathbf{0}$ & & 1 & & 2 & & & \\
\hline 'Hamlin' & 3 a 5 anos & 207,66 & $\mathrm{a}$ & 214,32 & a & 199,04 & $\mathrm{a}$ & $0,30^{\mathrm{NS}}$ & 24,25 \\
\hline 'Hamlin’' & 6 a 10 anos & 168,76 & $\mathrm{c}$ & 237,90 & $\mathrm{~b}$ & 362,58 & $\mathrm{a}$ & $76,82 * *$ & 23,09 \\
\hline 'Hamlin’' & 11 a 15 anos & 247,20 & $\mathrm{~b}$ & 286,28 & $a b$ & 323,25 & $\mathrm{a}$ & $12,25 * *$ & 14,26 \\
\hline 'Pêra' & 3 a 5 anos & 203,95 & $\mathrm{a}$ & 242,70 & a & 205,40 & $\mathrm{a}$ & $1,80^{\mathrm{NS}}$ & 20,29 \\
\hline 'Pêra' & 6 a 10 anos & 156,05 & $\mathrm{~b}$ & 205,05 & a & 221,63 & $\mathrm{a}$ & $27,26 * *$ & 23,63 \\
\hline 'Pêra' & 11 a 15 anos & 219,15 & $\mathrm{~b}$ & 277,88 & $a b$ & 359,28 & $\mathrm{a}$ & $11,66 * *$ & 19,00 \\
\hline 'Natal' & 3 a 5 anos & 200,38 & $\mathrm{a}$ & 197,90 & a & 211,18 & $\mathrm{a}$ & $0,15^{\mathrm{NS}}$ & 19,59 \\
\hline 'Natal' & 6 a 10 anos & 227,88 & $\mathrm{a}$ & 288,18 & $\mathrm{a}$ & 302,28 & $\mathrm{a}$ & $4,79^{\mathrm{NS}}$ & 22,99 \\
\hline 'Natal' & 11 a 15 anos & 206,43 & $\mathrm{c}$ & 260,13 & $\mathrm{~b}$ & 313,48 & $\mathrm{a}$ & $22,94 * *$ & 19,41 \\
\hline 'Valência' & 3 a 5 anos & 172,78 & $\mathrm{a}$ & 179,83 & $\mathrm{a}$ & 164,02 & $\mathrm{a}$ & $0,34^{\mathrm{NS}}$ & 21,36 \\
\hline 'Valência' & 6 a 10 anos & 195,73 & $\mathrm{~b}$ & 268,33 & $a b$ & 329,25 & $\mathrm{a}$ & $10,73 *$ & 17,29 \\
\hline 'Valência' & 11 a 15 anos & 222,10 & $\mathrm{c}$ & 277,48 & $\mathrm{~b}$ & 376,30 & $\mathrm{a}$ & $223,24 * *$ & 15,97 \\
\hline
\end{tabular}

${ }^{1}$ Valores com a mesma letra na linha não diferem significativamente entre si pelo Teste de Tukey ao nível de 5\% de probabilidade;

${ }^{2}$ Valor de F não significativo (NS), significativo ao nível de $5 \%(*)$ ou $1 \%(* *)$ de probabilidade.

causa o "anelamento" ao redor de todo tronco e, como conseqüência, prejudica o transporte dos produtos sintetizados pela fotossíntese nas folhas da copa para as raízes e radicelas. O fluxo restrito de seiva elaborada para as raízes e radicelas por um período de tempo prolongado, por sua vez, acaba por exaurir as reservas de carboidratos das mesmas, favorecendo o ataque de patógenos secundários e causando a morte das raízes e radicelas por desnutrição (Bassanezi et al., 2003b). À medida que o sistema radicular da planta afetada é diminuído, a absorção de água e sais minerais pelas raízes e radicelas também é prejudicada. O desequilíbrio entre a demanda da copa por água e sais minerais e o suprimento fornecido pelo sistema radicular é, então, responsável pelos sintomas de definhamento generalizado observados na copa, como a palidez e murcha das folhas, ausência de novas brotações vigorosas e queda de folhas (Gimenes-Fernandes \& Bassanezi, 2001).

Plantas cítricas com estresse hídrico e nutricional e com menor índice de área foliar apresentam menor eficiência fotossintética e, conseqüentemente, menor capacidade de acúmulo de carboidratos (Syvertsen \& Lloyd, 1994). Uma vez que as condições ambientais necessárias para a indução do florescimento e frutificação tenham sido satisfeitas, a condição e a disponibilidade das reservas de carboidratos na planta é uma das mais importantes limitações para a produção de frutos cítricos (Goldschmidt et al., 1985; Garcia-Luis et al., 1988; Davenport, 1990). Quanto maior a disponibilidade de carboidratos na planta e a quantidade de açúcares nos frutos, maior será o número de flores e menor será a queda dos frutos, isto é, maior o pegamento dos frutos e, conseqüentemente, maior o número de frutos que serão colhidos na planta (Guardiola, 2000). O crescimento e o tamanho final dos frutos resultam do acúmulo de matéria seca e água, que depende da competição entre os frutos por metabólitos e da disponibilidade destes metabólitos na árvore, além da capacidade do sistema radicular em absorver água e sais minerais (Fishler et al., 1983; Guardiola, 1992).

A ausência de diferença entre as variedades em relação aos danos causados pela MSC na produção, sugere que as variedades de laranjeira doce apresentam o mesmo nível de tolerância à doença em relação às demais, ou que estes danos dependem exclusivamente das alterações provocadas pela MSC no porta-enxerto e da capacidade do câmbio vascular do porta-enxerto recuperar os floemas degradados, permitir a passagem de fotoassimilados para as raízes e restabelecer equilíbrio entre o sistema radicular e a copa.

Plantas maiores, com mais folhas e mais produtivas apresentam maior quantidade de carboidratos armazenados que plantas mais novas, cujo consumo de reservas para o crescimento vegetativo é maior. Portanto, o maior pegamento dos frutos em plantas mais velhas é maior que em plantas mais novas. Já, o desequilíbrio entre a demanda e o suprimento de água e sais minerais nas plantas acima de cinco anos afetadas pela MSC é maior que nas plantas jovens, apresentando, então, menor tamanho dos frutos. Freqüentemente, o tamanho dos frutos cítricos mostra uma relação inversa com o número de frutos na árvore (Goldschmidt \& Monselise, 1977; Guardiola, 1988).

Após a constatação das primeiras plantas com sintomas de MSC, a doença pode se manifestar em 60 a $100 \%$ das plantas do talhão em até dois anos (Bassanezi et al., 2003b). Contudo, na maioria dos casos, durante este mesmo período, a maioria das plantas sintomáticas se encontra entre os níveis 1 e 2 de severidade (dados não publicados). Com base nos resultados deste trabalho, poder-se-á estimar a viabilidade econômica do talhão antes que medidas de erradicação sejam tomadas, considerando-se a produtividade média esperada para as plantas sadias e em cada nível de 
severidade de MSC, o custo de produção por caixa, o valor pago por caixa e o número de plantas nos diferentes níveis de sintomas de MSC.

\section{REFERÊNCIAS BIBLIOGRÁFICAS}

BASSANEZI, R.B., BERGAMIN FILHO, A., AMORIM, L., GIMENES-FERNANDES, N., GOTTWALD, T.R. \& BOVÉ, J.M. Spatial and temporal analyses of citrus sudden death as a tool to generate hypotheses concerning its etiology. Phytopathology 93:502512. 2003a.

BASSANEZI, R.B., GIMENES-FERNANDES, N. \& YAMAMOTO, P.T. Morte Súbita dos Citros. Araraquara: Fundecitrus/EECB, Boletim Citrícola, 24. 2003b.

BASSANEZI, R.B., YAMAMOTO, P.T. \& GIMENESFERNANDES, N. Progresso dos sintomas de "morte súbita” em pomares de laranjeiras 'Valência' e 'Pêra'. Summa Phytopathologica 28:23. 2002. (Resumo)

DAVENPORT, T.L. Citrus flowering. Horticultural Review 12:349408, 1990.

FISHLER, M., GOLDSCHMIDT, E.E. \& MONSELISE, S.P. Leaf area and fruit size in girdled grapefruit branches. Journal of American Society of Horticultural Science 108:218-221. 1983.

FUNDECITRUS. MSC evolui. Revista do Fundecitrus 120:6-7. 2004.

GARCIA-LUIS, A., FERNES, F., SANZ, A. \& GUARDIOLA, J.J. The regulation of flowering and fruit set in Citrus: relationship with carbohydrate level. Israel Journal of Botany 37:189-201. 1988. GIMENES-FERNANDES, N. \& BASSANEZI, R.B. Doença de causa desconhecida afeta pomares cítricos no norte de São Paulo e sul do Triângulo Mineiro. Summa Phytopathologica 27:93. 2001.

GOLDSCHMIDT, E.E., ASHKEMAZI, M., HERZANO, Y., SHAFFER, A.A. \& MONSELISE, S.P. A role for carbohydrate levels in the control of flowering in citrus. Scientia Horticulturae 26:159166. 1985.

GOLDSCHMIDT, E.E. \& MONSELISE, S.P. Physiological assumptions toward the development of a citrus fruiting model. Proceedings of International Society of Citriculture 2:668-672. 1977. GUARDIOLA, J.L. Factors limiting productivity in citrus: a physiological approach. Proceedings, 6th International Citrus Congress. 1988. pp.381-394.

GUARDIOLA, J.L. Frutificação e crescimento. In: Donadio, L.C. (Coord.). Anais do Segundo Seminário Internacional de Citros: Fisiologia. Bebedouro, Fundação Cargill, 1992. pp.1-24.

GUARDIOLA, J.L. Regulation of flowering and fruit development: endogenous factors and exogenous manipulation. Proceedings, 9th International Citrus Congress. 2000. pp.342-346.

JESUS JUNIOR, W.C. \& BASSANEZI, R.B. Análise da dinâmica e estrutura de focos da morte súbita dos citros. Fitopatologia Brasileira 29:399-405. 2004.

KIMATI, H. \& GALLI, F. Doenças dos citros. In: Galli, F. (Coord.) Manual de Fitopatologia: doenças das plantas cultivadas v.2. São Paulo. Ed. Agronômica Ceres. 1980. pp.213-235.

LIBANORE, A.C., MASSARI, C.A., BASSANEZI, R.B., YAMAMOTO, P.T., GIMENES-FERNANDES, N. \& AYRES, A.J. Levantamento da ocorrência da "morte súbita dos citros" no Estado de São Paulo e sul do Triângulo Mineiro. Summa Phytopathologica 28:72-73. 2002. (Resumo)

MÜLLER, G.W., DE NEGRI, J.D., AGUILAR-VILDOSO, C.I., MATTOS JR., D., POMPEU JR., J., TEÓFILO SOBRINHO, J., CARVALHO, S.A., GIROTTO, L.F. \& MACHADO, M.A. Morte súbita dos citros: uma nova doença na citricultura brasileira. Laranja 23:371-386. 2002.

ROMÁN, M.P., CAMBRA, M., JUÁREZ, J., MORENO, P., DURAN-VILA, N., TANAKA, F.O.A., ALVES, E., KITAJIMA, E.W., YAMAMOTO, P.T., BASSANEZI, R.B., TEIXIERA, D.C., JESUS JUNIOR, W.C., AYRES, A.J., GIMENES-FERNANDES, N., RABENSTEIN, F., GIROTTO, L.F. \& BOVÉ, J.M. Sudden death of citrus in Brazil: a graft-transmissible bud union disease. Plant Disease 88:453-467. 2004.

SYVERTSEN, J.P. \& LLOYD, J.J. Citrus. In: Schaffer, B. \& Anderson, P.C. (Eds.) Handbook of environmental physiology of fruit crops. CRC Press, Boca Raton, 1994. v.2, pp.65-99.

YAMAMOTO, P.T., JESUS JUNIOR, W.C., BASSANEZI, R.B., SANCHES, A.L., AYRES, A.J., GIMENES-FERNANDES, N. \& BOVÉ, J.M. Transmission of the agent inducing symptoms of citrus sudden death by graft-inoculation under insect-proof conditions. Fitopatologia Brasileira 28:S265. 2003. (Resumo) 